\title{
PIGD: a database for intronless genes in the Poaceae
}

\author{
Hanwei Yan, Cuiping Jiang, Xiaoyu Li, Lei Sheng, Qing Dong, Xiaojian Peng, Qian Li, Yang Zhao, \\ Haiyang Jiang and Beijiu Cheng*
}

\begin{abstract}
Background: Intronless genes are a feature of prokaryotes; however, they are widespread and unequally distributed among eukaryotes and represent an important resource to study the evolution of gene architecture. Although many databases on exons and introns exist, there is currently no cohesive database that collects intronless genes in plants into a single database.

Description: In this study, we present the Poaceae Intronless Genes Database (PIGD), a user-friendly web interface to explore information on intronless genes from different plants. Five Poaceae species, Sorghum bicolor, Zea mays, Setaria italica, Panicum virgatum and Brachypodium distachyon, are included in the current release of PIGD. Gene annotations and sequence data were collected and integrated from different databases. The primary focus of this study was to provide gene descriptions and gene product records. In addition, functional annotations, subcellular localization prediction and taxonomic distribution are reported. PIGD allows users to readily browse, search and download data. BLAST and comparative analyses are also provided through this online database, which is available at http://pigd.ahau.edu.cn/.
\end{abstract}

Conclusion: PIGD provides a solid platform for the collection, integration and analysis of intronless genes in the Poaceae. As such, this database will be useful for subsequent bio-computational analysis in comparative genomics and evolutionary studies.

Keywords: Poaceae, Intronless Genes, Database

\section{Background}

In eukaryotes, most genes are composed of exons and introns. Exons are the coding regions of genes that are transcribed and collectively form a contiguous coding sequence. Introns, commonly found in eukaryotes between exon sequences in the DNA, are removed from RNA molecules through splicing during post-transcriptional RNA processing. Although the exon-intron gene structure, the split structure of genes, is typical, intronless or singleexon genes account for a significant proportion in most eukaryotes. Intronless genes are a characteristic feature of prokaryotes and an important resource for understanding the rules of gene structure and organization, protein functionality and evolutionary differences between eukaryotes and prokaryotes. With the availability of complete genome

\footnotetext{
* Correspondence: bjchengahau@163.com

Key Laboratory of Crop Biology of Anhui Province, Anhui Agricultural University, Hefei 230036, China
}

sequences, several studies have attempted to identify intronless genes in many eukaryotic genomes and subsequently determine their functional and evolutionary history. To date, 2,017 ( 8\%) intronless genes have been identified in mice [1]; 930-6,229 (7.9\%-16.7\%) in deuterostomes [2]; 542-1,014 (2.8\%-4.5\%) in teleost fish [3]; 11,109 (19.9\%) in rice; and 5,846 (21.7\%) in Arabidopsis thaliana [4]. These intronless genes encode proteins from a variety of families, including G-protein-coupled receptors (GPCRs) [5,6], olfactory receptors $[7,8]$ and small auxin-up RNAs (SAURs) [9], which are essential for various biological functions. Data show that proteins encoded by intronless genes participate in growth regulation, cell proliferation, development, sperm formation and immune responses [10-12]. The origin of intronless genes is not clear and represents a fascinating area of research, partly because of their prokaryote-like structure. It has been suggested that intronless genes form when introns are lost; this would generate a subset of intron-free genes [13]. 
Another possibility is that intronless genes evolved as a result of reverse transcription [14]. In the process of retroposition, mRNAs are reverse-transcribed into cDNA and inserted into new genomic positions that lack introns [15].

To date, three intronless gene databases have been created, including SEGE for Homo sapiens, Drosophila melanogaster, Saccharomyces cerevisiae, Caenorhabditis elegans and Arabidopsis thaliana genes [16]; Genome SEGE for eukaryotic genome sequences from NCBI [17]; and IGD for human intronless genes [18]. Currently, IGD is the only intronless gene database available on the Internet. Until now, no intronless gene database has been developed for sequenced Poaceae genomes.

With this in mind, we present the Poaceae Intronless Genes Database (PIGD; http://pigd.ahau.edu.cn/). This database, with a user-friendly web interface, provides access to a collection of intronless genes from five genome sequenced Poaceae, including Sorghum bicolor, Zea mays, Setaria italica, Panicum virgatum and Brachypodium distachyon. PIGD will allow researchers to easily identify intronless genes, integrate functional and evolutionary annotations, and search and download detailed information. In contrast to databases dedicated to individual organisms, the PIGD provides comparative analyses of genomic data from five species, and in conjunction with the BLAST program, it will allow users to explore and analyze data generated from the PIGD efficiently. We anticipate that PIGD will become a useful resource for the research community, particularly for studies of molecular function and the evolution of intronless genes.

\section{Construction and content}

\section{Data sources}

Currently, PIGD includes the following five organisms Sorghum bicolor, Zea mays, Setaria italica, Panicum virgatum and Brachypodium distachyon. All genome data were downloaded from the Phytozome database (release version 9.1, http://www.phytozome.net/IEsorry.php?refer=/) [19].

\section{Identification of Poaceae intronless genes}

A stringent protocol was used to reliably identify Poaceae intronless genes. First, using the Java program, we extracted genes that contain a "CDS" line in the GFF3 format files. Redundant sequences representing the same gene loci were then excluded. Finally, we retained sequences assembled into chromosomes for Sorghum bicolor, Zea mays and Brachypodium distachyon. Organelle sequences were discarded to avoid ambiguity. Scaffolds that could not be physically anchored in Setaria italica and Panicum virgatum were present in the data set. Thus, lists of non-redundant intronless genes from the five Poaceae organisms were generated.

\section{Data assembly}

Each entry into PIGD integrates a comprehensive list of annotations on: (i) physical location, strand, protein length from Phytozome; (ii) gene family from Pfam [20] (http://pfam.sanger.ac.uk/); (iii) isoelectric point (PI) and molecular weight (Mw) from Expasy [21] (http://www. expasy.org/); (iv) GI number and definition from the NCBI protein project (http://www.ncbi.nlm.nih.gov/protein/); (v) a prediction of function based on cellular role and Gene Ontology (GO) from Protfun [22] (http://www.cbs.dtu.dk/ services/ProtFun/); (vi) prediction of subcellular localization from WoLF PSORT [23] (http://www.genscript. com/psort/wolf_psort.html) and (vii) taxonomic distribution among different groups (archaea, bacteria, metazoans, fungi, plants, viruses and other eukaryotes) using Blink [24] (http://www.ncbi.nlm.nih.gov/sutils/blink. cgi? mode= query).

\section{Comparisons between Poaceae species}

The records contained within the "Comparison" menu are grouped into eight general classes of related information: (i) the number and percentage of intronless genes; (ii) the distribution of PI; (iii) the distribution of $\mathrm{Mw}$; (iv) the distribution of protein length; (v) the distribution of cellular roles; (vi) the distribution of GO categories; (vii) the number and percentage of species-specific intronless genes; and (viii) the gene size of the species-specific intronless genes.

\section{The PIGD web interface}

A web-based platform, PIGD combined the MySQL (version 5.5.8) database management system with a dynamic web interface based on PHP (version 5.3.3) and Javascript (version 1.2).

\section{Utility}

The PIGD currently contains 54,336 intronless genes from five sequenced Poaceae species (Table 1). Protein and transcript sequences were downloaded from Phytozome. The lack of comprehensive and consistent intronless gene databases means that it was essential to integrate information from all possible sources. Hence, we chose several representative data sources and analytic tools, including Pfam, Expasy, NCBI, Protfun and WoLF PSORT,

Table 1 The number of intronless genes reported for each species

\begin{tabular}{lc}
\hline Species & The number of intronless genes \\
\hline Sorghum bicolor & 6,197 \\
Zea mays & 14,623 \\
Setaria italica & 10,015 \\
Panicum virgatum & 17,227 \\
Brachypodium distachyon & 6,274 \\
\hline
\end{tabular}


to help aid the overall understanding of the function and evolution of intronless genes. Intronless gene sequences and annotation data were stored in a MySQL relational database for efficient retrieval of data from indexed files.

The web interface of PIGD was designed to include the following components: Home, Search, BLAST, Browse (for the database), Comparison, Download and Help. An illustration of the PIGD system is shown in Figure 1.

\section{Home}

The user is introduced to the objective of the PIGD, the major scientific contributions, and the species that are currently included in the database. Details about the organization that developed the PIGD and contact information are also provided at the top of the home page.

\section{Search}

There are two different ways to search the database: quick and advanced searches. Users can either type a truncated version or the entire ID for a gene, transcript and protein and PIGD identifier into the search field found on the top right of each page. Alternatively, an advanced search can be conducted, where the user is able to assign the species, chromosome, cellular role, GO category and Gene ID of interest, by clicking on the "Search" button on the home page. The results pages matching the query conditions are then shown. Finally, users are able to easily navigate from their search results to pages that contain the detailed annotations.

\section{Blast}

BLAST search (release version 2.2.9) can be conducted from http://pigd.ahau.edu.cn/BLAST.php to search PIGD using DNA or protein sequence(s) as the query(s). Users can apply five BLAST search programs (blastn, blastp, blastx, tblastn and tblastx) with an E-value $\leq 1 \mathrm{e}-5$ to find the putative homologous sequences of these intronless genes in different species.

\section{Browse}

From the home page, users can click on "Browse" to select their desired species from the drop-down menu or by clicking directly on the image of the species seen in the middle of the home page. A page providing general information, including an overview of the species, the number of intronless genes, and chromosomal distribution, is then shown. In addition, functional categorization (cellular role and GO category); classification of intronless genes into the three super kingdoms of life (archaea, bacteria and eukaryotes); the distribution of PI, Mw and protein length; and the sources of all primary gene annotations are provided. Users are then able to click on part of the image from the "Browse" page to open a new window with gene entries that meet the specific conditions. An individual gene annotation displays basic information related to the gene description, including the physical location and chromosome strand and gene product. Gene product records contain the protein length, gene family attribute, PI, Mw, GI number and definition on NCBI, and mRNA coding and protein sequences. In addition, functional assignment, subcellular localization prediction and taxonomic distribution among different groups (archaea, bacteria, metazoans, fungi, plants, viruses and other eukaryotes) are provided for further study of the functional and evolutionary patterns of intronless genes. Some gene features are hyperlinked to their respective gene pages and to external sites and databases that provide more detailed information. In general, a cascading style is applied for data browsing, which is browsed in steps, starting with species, then providing an intronless gene list and finally single gene annotations (Figure 1).

\section{Comparison}

The results of comparative analysis among the five species are shown on Comparison interface. A comprehensive analysis of the genomes of Sorghum bicolor, Zea mays, Setaria italica, Panicum virgatum and Brachypodium distachyon showed that these consist of $22.45 \%(6,197)$, $36.87 \%(14,623), 28.23 \%(10,015), 26.15 \%(17,227)$ and $23.63 \%(6,274)$ intronless genes, respectively (Figure 2A). The variability in the number of intronless genes observed is clearly correlated with the size of the genome. A bimodal distribution of PI in proteins encoded by intronless genes was observed in all five Poaceae species, with a smaller third peak between two main peaks (Figure 2B). These results are consistent with those previously reported [25]. The distribution of the $\mathrm{Mw}$ of intronless genes was unimodal, with a peak value ranging from 7 to $13 \mathrm{kDa}$ (Figure 2C). To investigate the general trends in protein length distribution, five scatter plots of protein length were generated (Figure 2D). The average protein length was approximately 337 amino acids (aa) in Sorghum bicolor, 222 aa in Zea mays, 215 aa in Setaria italica, 249 aa in Panicum virgatum and 331 aa in Brachypodium distachyon. To explore the biological function of proteins encoded by intronless genes, the cellular roles and GO categories of these genes were predicted and compared in each species. The results showed that the percentages of intronless genes that fell under the different functional categories were similar across species, with the largest number of intronless genes categorized as translation (T) and growth factor (GF) in the cellular role and GO categories, respectively (Figure 2E, F). In addition, the genic parameters for species-specific intronless genes were calculated, including gene number and length (Figure 2G, H). Overall, the comparative analyses of intronless genes among Poaceae species clearly showed several common functional and evolutionary characteristics 


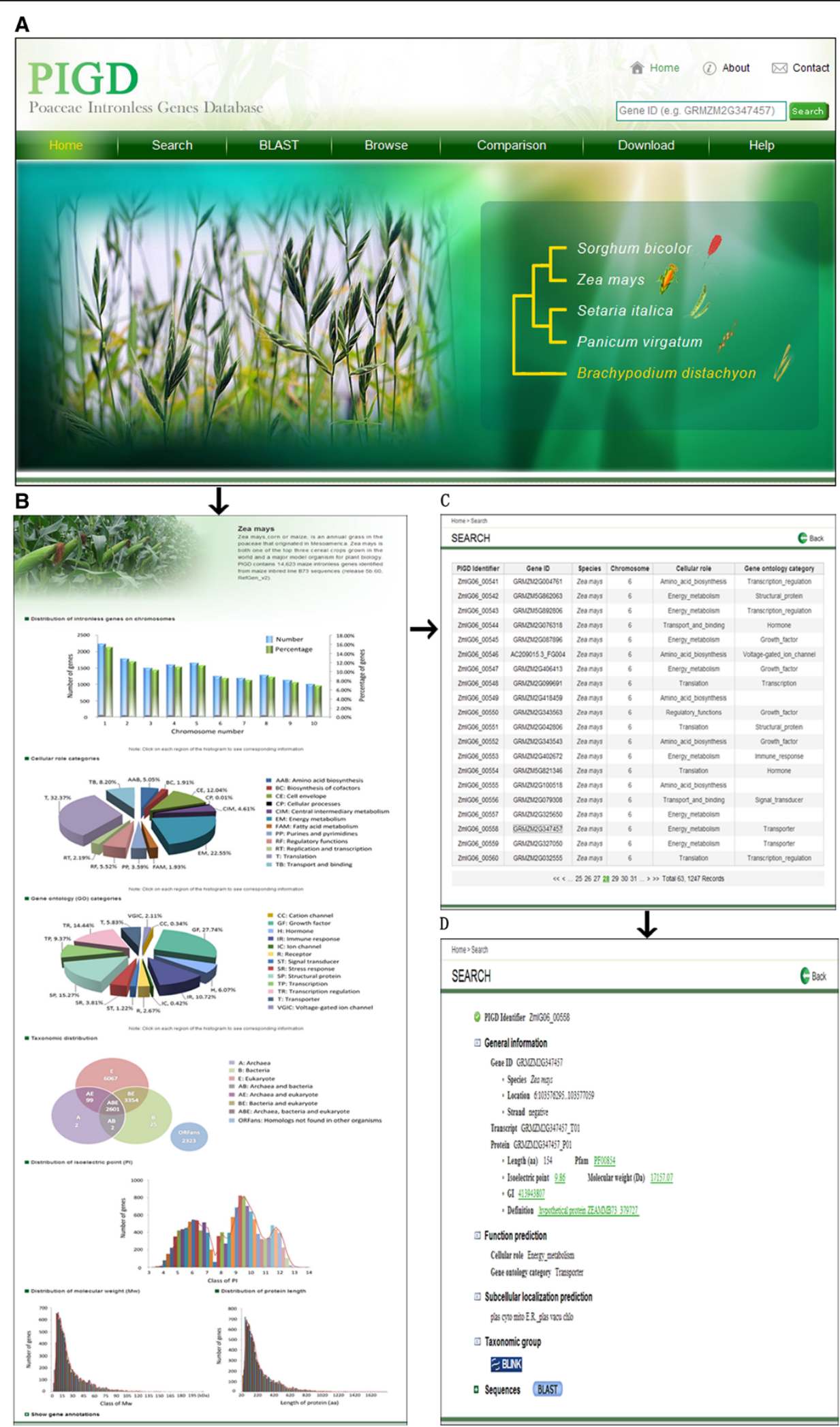

Figure 1 An overview of the website and gene annotation page in the PIGD. (A) The Home page. (B) The summary data for intronless genes found in Zea mays. (C) An example list of intronless genes that can be produced for a specific organism. (D) An example of the gene annotation page. 


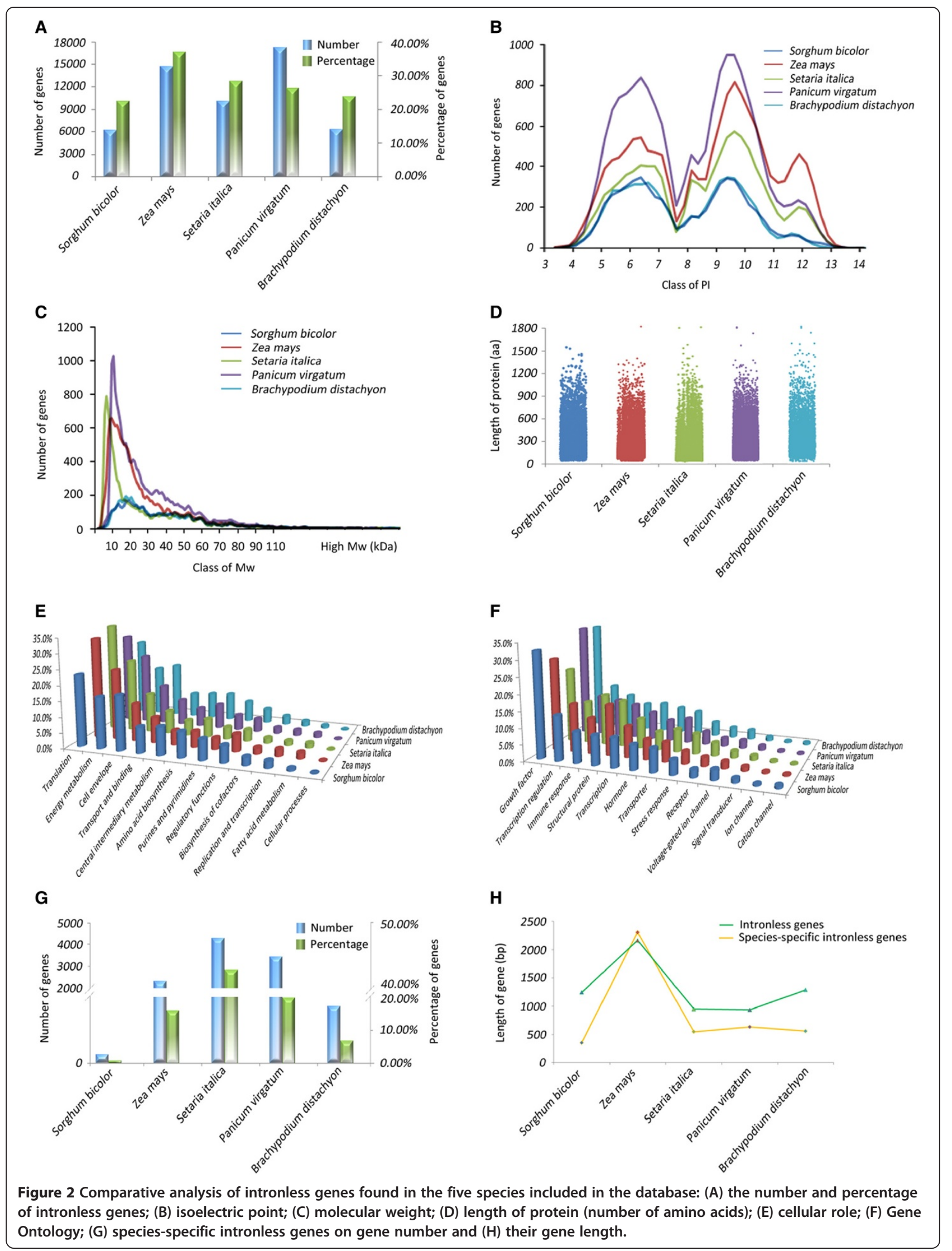


that may also be found in other major eukaryote kingdoms. However, species-specific features remain and may represent one of the sources of the observed natural biodiversity.

\section{Download}

A file transfer protocol (FTP) download server provides users the option to download files in bulk form.

\section{Help}

To help address any specific problems or issues users may encounter, a detailed tutorial was developed. This tutorial introduces the user to the PIGD interface and is accessed from the "Help" drop-down menu. In addition, links to external databases have been provided. PIGD has a data submitting system via which we encourage researchers to submit data about intronless genes in other eukaryotic genomes. The database administrators will approve sequences and annotation information submitted by users before their inclusion into PIGD.

\section{Discussion}

Recently, eukaryotic intronless genes have gained increasing attention because of their potential use in understanding evolutionary patterns of related genes and genomes. Relevant databases have been set up and have aided evolutionary and functional studies. For example, researchers identified six 10-nt motifs that are present in more than $90 \%$ of the intronless genes, using the IGD data. It is further hypothesized that one or more of these motifs function in intronless mRNA export and/or that a structure is involved [26]. However, to date, there is no such dedicated database for intronless genes in plants. Given the rapid advancement of next-generation sequencing, more and more plant genomes are being sequenced. Developing a plant intronless genes database could greatly aid research by data mining such valuable genomic resources. In PIGD, we offer a feature-rich and user-friendly integration of data, tools and analyses.

First, PIGD provides comparative genomic data for the Poaceae that permits the identification of features that are conserved or divergent during evolution (Figure 2). We used the characterization of species-specific intronless genes as an example. The study of species-specific genes has generated much interest in recent years because these genes are of particular importance for understanding plant adaptation. Comparative analysis of species-specific intronless genes showed the dramatic difference on their gene number among the five species. Meanwhile, Yang et al. [27] compared specific genes identified in plants and animals, and found that there are many more specific genes in plant genomes. This difference may suggest that gene duplication followed by rapid evolution occurred in plants at a higher frequency [28]. Detailed information on species-specific intronless genes makes it possible for the user to investigate codon usages, syntenic genomic regions and molecular comparison, thereby help the user to determine the exact mechanisms regarding the origin of species-specific genes.

Second, popular tools such as NCBI BLAST are embedded in our website to help annotate new sequences and predict putative orthologous relationships of genes. Also, PIGD provides an interactive platform for communication and data sharing among members of the same research team, where researchers can download the available data and submit their important scientific findings.

The PIGD database will be updated at least once a year, and the number of available plant species included will be expanded. Future versions of the database are expected to include detailed gene annotations, such as RNA-seq data, paralogs and KEGG ontology, to enable researchers to further explore the function and evolution of intronless genes. Meanwhile, powerful comparative analysis tools of intronless genes in plants will be developed to provide a centralized platform that could benefit a wide variety of related research.

\section{Conclusion}

PIGD is the only existing plant intronless genes database that complements the IGD database for human intronless genes. The availability of online sequence databases provides an easy entry point for researchers to immediately access information about intronless genes, without the need to install additional software. Furthermore, statistical analyses relevant to genetic features and functional classification systems are provided in both databases. Such integration and analysis of data may help users understand the biological role of intronless genes in the genomes of higher organisms and explore the functionality of introns in gene evolution.

\section{Availability and requirements}

PIGD is freely accessible at http://pigd.ahau.edu.cn/. Moreover, all data are available for download in flat file form from the FTP sites. Inquiries concerning the database may be directed to PIGD_MKL@163.com. Operating system(s): Platform independent.Programming languages: PHP, MySQL, HTML and JavaScript. License: Not required. Any restrictions to use by non-academics: None.

\section{Abbreviations}

PIGD: Poaceae Intronless Genes Database; GPCRs: G-protein-coupled receptors; SAURs: Small auxin-up RNAs; PI: Isoelectric point; Mw: Molecular weight; GO: Gene Ontology; T: Ttranslation; GF: Growth factor.

\section{Competing interests}

The authors declare that they have no competing interests. 


\section{Authors' contributions}

$\mathrm{HY}$ and $\mathrm{CJ}$ performed the integration, constructed the database platform, and wrote the manuscript. $L X, L S, Q D, X P, Q L$ and $Y Z$ helped to design the database platform and update the database, and provided scientific suggestions and criticisms for improving the manuscript and the website. $\mathrm{HJ}$ and BC participated in the design, helped write the manuscript and supervised the whole project. All authors read and approved the final manuscript.

\section{Acknowledgments}

The authors are grateful for the support from the Genetically Modified Organisms Breeding Major Projects (Grant No. 2013ZX08003-002), and the National Natural Science Foundation of China (Grant Nos. 11075001, 31101159, 31201217).

Received: 30 June 2014 Accepted: 24 September 2014 Published: 1 October 2014

\section{References}

1. Sakharkar KR, Sakharkar MK, Culiat CT, Chow VT, Pervaiz S: Functional and evolutionary analyses on expressed intronless genes in the mouse genome. FEBS Lett 2006, 580(5):1472-1478.

2. Zou M, Guo B, He S: The roles and evolutionary patterns of intronless genes in deuterostomes. Comp Funct Genomics 2011, 28(39):1-8.

3. Tine $M$, Kuhl H, Beck A, Bargelloni L, Reinhardt R: Comparative analysis of intronless genes in teleost fish genomes: insights into their evolution and molecular function. Mar Genomics 2011, 4(2):109-119.

4. Jain M, Khurana P, Tyagi AK, Khurana JP: Genome-wide analysis of intronless genes in rice and Arabidopsis. Funct Integr Genomics 2008, 8(1):69-78.

5. Gentles AJ, Karlin S: Why are human G-protein-coupled receptors predominantly intronless? Trends Genetics 1999, 15(2):47-49.

6. Takeda S, Kadowaki S, Haga T, Takaesu H, Mitaku S: Identification of G protein-coupled receptor genes from the human genome sequence. FEBS Lett 2002, 520(1-3):97-101.

7. Zhang $X$, Firestein S: The olfactory receptor gene superfamily of the mouse. Nat Neurosci 2002, 5(2):124-133.

8. Lecharny A, Boudet N, Gy I, Aubourg S, Kreis M: Introns in, introns out in plant gene families: a genomic approach of the dynamics of gene structure. J Struct Funct Genomics 2003, 3(1-4):111-116.

9. Jain M, Tyagi AK, Khurana JP: Genome-wide analysis, evolutionary expansion, and expression of early auxin-responsive SAUR gene family in rice (Oryza sativa). Genomics 2006, 88(3):360-371.

10. Markovic D, Challiss RA: Alternative splicing of $G$ protein-coupled receptors: physiology and pathophysiology. Cell Mol Life Sci 2009, 66(20):3337-3352

11. Dorsam RT, Gutkind JS: G-protein-coupled receptors and cancer. Nat ReV Cancer 2007, 7(2):79-94.

12. Grzybowska EA: Human intronless genes: functional groups, associated diseases, evolution, and mRNA processing in absence of splicing. Biochem Biophys Res Commun 2012, 424(1):1-6.

13. Catania F, Lynch M: Where do introns come from? PLoS Biol 2008 , 6(11):e283.

14. Zhu Z, Zhang $Y$, Long M: Extensive structural renovation of retrogenes in the evolution of the Populus genome. Plant Physiol 2009, 151(4):1943-1951.

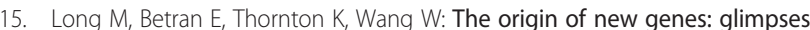
from the young and old. Nat Rev Genet 2003, 4(11):865-875.

16. Sakharkar MK, Kangueane P, Petrov DA, Kolaskar AS, Subbiah S: SEGE: A database on 'intron less/single exonic' genes from eukaryotes. Bioinformatics 2002, 18(9):1266-1267.

17. Sakharkar MK, Kangueane P: Genome SEGE: a database for 'intronless' genes in eukaryotic genomes. BMC Bioinformatics 2004, 5:67.

18. Louhichi A, Fourati A, Rebai A: IGD: a resource for intronless genes in the human genome. Gene 2011, 488(1-2):35-40

19. Goodstein DM, Shu S, Howson R, Neupane R, Hayes RD, Fazo J, Mitros T, Dirks W, Hellsten U, Putnam N, Rokhsar DS: Phytozome: a comparative platform for green plant genomics. Nucleic Acids Res 2011, 40(Database issue):D1178-1186.

20. Punta M, Coggill PC, Eberhardt RY, Mistry J, Tate J, Boursnell C, Pang N, Forslund K, Ceric G, Clements J, Punta M, Coggill PC, Eberhardt RY, Mistry J,
Tate J, Boursnell C, Pang N, Forslund K, Ceric G, Clements J, Heger A, Holm L, Sonnhammer ELL, Eddy SR, Bateman A, Finn RD: The Pfam protein families database. Nucleic Acids Res 2012, 40(Database issue):D290-301.

21. Artimo $P$, Jonnalagedda M, Arnold K, Baratin D, Csardi G, de Castro E, Duvaud S, Flegel V, Fortier A, Gasteiger E, Grosdidier A, Hernandez C, loannidis V, Kuznetsov D, Liechti R, Moretti S, Mostaguir K, Redaschi N, Rossier G, Xenarios I, Stockinger H: ExPASy: SIB bioinformatics resource portal. Nucleic Acids Res 2012, 40(Web Server issue):W597-603.

22. Jensen LJ, Gupta R, Staerfeldt HH, Brunak S: Prediction of human protein function according to Gene Ontology categories. Bioinformatics 2003, 19(5):635-642.

23. Horton P, Park KJ, Obayashi T, Fujita N, Harada H, Adams-Collier CJ, Nakai K: WoLF PSORT: protein localization predictor. Nucleic Acids Res 2007, 35(Web Server issue):W585-587.

24. Wheeler DL, Barrett T, Benson DA, Bryant SH, Canese K, Church DM DiCuccio M, Edgar R, Federhen S, Helmberg W, Kenton DL, Khovayko O, Lipman DJ, Madden TL, Maglott DR, Ostell J, Pontius JU, Pruitt KD, Schuler GD, Schriml LM, Sequeira E, Sherry ST, Sirotkin K, Starchenko G, Suzek TO, Tatusov R, Tatusova TA, Wagner L, Yaschenko E: Database resources of the National Center for Biotechnology Information. Nucleic Acids Res 2005, 33(Database issue):D39-45.

25. Weiller GF, Caraux G, Sylvester N: The modal distribution of protein isoelectric points reflects amino acid properties rather than sequence evolution. Proteomics 2004, 4(4):943-949.

26. Lei H, Zhai B, Yin S, Gygi S, Reed R: Evidence that a consensus element found in naturally intronless mRNAs promotes mRNA export. Nucleic Acids Res 2013, 41(4):2517-2525.

27. Yang L, Zou M, Fu B, He S: Genome-wide identification, characterization, and expression analysis of lineage-specific genes within zebrafish. BMC Genomics 2013, 14:65.

28. Udall JA, Wendel JF: Polyploidy and crop improvement. Crop Sci 2006, 46:S3-S14.

doi:10.1186/1471-2164-15-832

Cite this article as: Yan et al:: PIGD: a database for intronless genes in the Poaceae. BMC Genomics 2014 15:832.

\section{Submit your next manuscript to BioMed Central and take full advantage of:}

- Convenient online submission

- Thorough peer review

- No space constraints or color figure charges

- Immediate publication on acceptance

- Inclusion in PubMed, CAS, Scopus and Google Scholar

- Research which is freely available for redistribution 